\title{
Chromosome 10q26 deletion syndrome: Two new cases and a review of the literature
}

\author{
SHAOBIN LIN*, YI ZHOU*, QUN FANG, JIANZHU WU, ZHIQIANG ZHANG, YUANJUN JI and YANMIN LUO \\ Fetal Medicine Center, Department of Obstetrics \& Gynecology, The First Affiliated Hospital of Sun Yat-Sen University, \\ Guangzhou, Guangdong 510080, P.R. China
}

Received September 29, 2015; Accepted October 6, 2016

DOI: $10.3892 / \mathrm{mmr} .2016 .5864$

\begin{abstract}
The current study presents the cases of two unrelated patients with similar clinical features, including craniofacial anomalies, developmental delay/intellectual disability and cardiac malformations, that are consistent with chromosome 10q26 deletion syndrome. High-resolution single-nucleotide polymorphism analysis revealed that $10 \mathrm{q} 26$ terminal deletions were present in these two patients. The locations and sizes of the 10q26 deletions in these two patients were compared with the locations and sizes of 10q26 deletions in 30 patients recorded in the DECIPHER database and 18 patients characterized in previous studies through chromosomal microarray analysis. The clinical features and locations of the 10q26 deletions of these patients were reviewed in an attempt to map or refine a critical region (CR) for phenotypes. Additionally, the association between previously suggested CRs and phenotypic variability was discussed. The current study emphasize that a distal 10q26 terminal deletion with a breakpoint at $\sim 130 \mathrm{Mb}$ may contribute to the common clinical features of $10 \mathrm{q} 26$ deletion syndrome.
\end{abstract}

\section{Introduction}

The partial deletion of distal chromosome $10 \mathrm{q}$ was first reported by Lewandowski et al (1), and the existence of a distinct chromosome 10q26 deletion syndrome (Online Mendelian Inheritance in Man no. 609625) was then suggested due to consistent clinical findings (2-4). This syndrome is caused by a rare chromosomal abnormality, although $\geq 110$ cases have been reported in the literature. Patients with

Correspondence to: Professor Yanmin Luo, Fetal Medicine Center, Department of Obstetrics \& Gynecology, The First Affiliated Hospital of Sun Yat-Sen University, 58 Zhongshan Road II, Guangzhou, Guangdong 510080, P.R. China

E-mail: luoyanm@mail.sysu.edu.cn

*Contributed equally

Key words: chromosome 10q26 deletion syndrome, critical region, genotype-phenotype correlation, SNP array this syndrome present with symptoms that span a relatively extensive and heterogeneous phenotypic spectrum, although common clinical features, including craniofacial anomalies, developmental delay/intellectual disability (DD/ID), urinary tract abnormalities, cardiac malformations and neurodevelopmental deficits, have been observed (4-7). A pure distal 10q deletion may be derived from familial or de novo structural variation and feature breakpoints at $10 \mathrm{q} 25$ or 10q26, although interstitial or terminal deletions of large size, which are typically identified through conventional cytogenetic techniques, can also occur (4,8). Chromosomal microarray analysis (CMA), including the use of array comparative genome hybridization and single-nucleotide polymorphism (SNP) arrays, has been recommended as the first-tier test for individuals with DD/ID and/or congenital anomalies (9). CMA has been increasingly employed to refine the location and size of 10q26 deletions to improve understanding of genotype-phenotype correlations by identifying the minimal critical region (CR) or the smallest region of deletion overlap associated with 10q26 deletion syndrome. Because this syndrome is a clinically heterogeneous disorder involving deletions of variable locations and sizes, the CR for 10q26 deletion syndrome and candidate genes for variable phenotypes remain unclear (10). Therefore, further studies on patients with $10 \mathrm{q} 26$ deletions should be performed to elucidate the correlation between various phenotypes and the CR. The majority of examined cases of this syndrome were previously characterized using traditional techniques with limited resolution and low efficiency, including G-banded karyotyping, fluorescence in situ hybridization and microsatellite marker genotyping, whereas few CMA-based molecular studies have been performed to investigate these cases. The current study attempted to refine a CR for this syndrome by performing a high-resolution molecular analysis of two unrelated patients with pure terminal 10q26 deletions and comparing these patients with patients with pure distal $10 \mathrm{q}$ deletions described in previous CMA-based studies and the Database of Chromosomal Imbalance and Phenotype in Humans using Ensembl Resources (DECIPHER; https://decipher.sanger.ac.uk/), specifically including patients from this database with deleted regions encompassed by the deleted regions of the two patients examined in the current study and that presented without any other copy number variants or with other likely benign copy number variants. In addition, the phenotypic variability associated with CRs proposed in previous studies is discussed. 


\section{Materials and methods}

Case presentation. Patient 1, a 3-year-old female, was the second child of healthy, unrelated parents. Prenatal ultrasonography indicated that duodenal atresia was present in the fetus; therefore, the karyotyping of fetal blood was performed. The fetal karyotype did not reveal any chromosomal abnormalities (data not shown). Thus, the patient's parents continued the pregnancy. Premature rupture of membranes occurred at 36 weeks of gestation. Eventually, the parents opted for delivery by caesarian section. No neonatal respiratory distress was observed. The infant's weight and length at birth were $2,420 \mathrm{~g}(<50$ th percentile) and $46 \mathrm{~cm}(<50$ th percentile), respectively. Surgical therapy for duodenal atresia was administered following birth, and an operation to repair patent ductus arteriosus (PDA) was performed at 12 days after birth. The child was referred to the Fetal Medicine Center (The First Affiliated Hospital of Sun Yat-Sen University, Guangzhou) due to DD and mild ID observed at 3 years and 9 months of age. Milestones were significantly delayed, with walking occurring at 3 years; current, the patient has no speech. The patient's psychomotor development was significantly delayed. At the age of 3 years and 9 months, her weight, height and head circumference were $9.6 \mathrm{~kg}$ ( $3 \mathrm{rd}$ percentile), $83.6 \mathrm{~cm}$ (3rd percentile) and $46.5 \mathrm{~cm}(<10$ th percentile), respectively. Cerebral magnetic resonance imaging did not reveal any abnormalities at this time. The patient exhibited certain craniofacial features of 10q26 deletion syndrome, including a triangular-shaped face, hypertelorism, strabismus, a broadly prominent nose and micrognathia. Palate anomalies were not observed. Her ear shape was normal, and audiometry indicated that she possessed normal hearing. An image of the patient is not available in the present report. Patient 2, a 5-year-old female, was the second child of healthy, unrelated parents. She was born at full term by caesarian section due to a scarred maternal uterus. Her birth weight and length were 2,450 g ( $<3$ rd percentile) and $47 \mathrm{~cm}(<10$ th percentile), respectively. Congenital heart defects (CHDs), including atrial septal defect and PDA, were observed by ultrasound examination during the neonatal period. At 3 months, the patient underwent an operation to address the CHDs. She walked and spoke her first word at 3 years. At the age of 5 years and 2 months, her weight, height and head circumference were $13 \mathrm{~kg}$ (3rd percentile), $96 \mathrm{~cm}$ (3rd percentile) and $47.6 \mathrm{~cm}$ (<10th percentile), respectively. The patient displayed normal motor skills and performed well at ice-skating at this time. However, mild ID and hyperactive behavior were observed. Craniofacial features of 10q26 deletion syndrome, including a high and narrow palatine arch, a triangular-shaped face, low-set ears, hypertelorism, strabismus, a broadly prominent nose and micrognathia, were observed. The patient's hearing was normal. Again, an image of the patient is not available in the present report.

Cytogenetic analysis. Using standard procedures, routine G-banded karyotyping was performed on peripheral blood specimens from each child and her parents, as previously described (11).

High-resolution SNP array analysis. Genomic DNA was isolated from peripheral blood lymphocytes with the QIAamp
DNA Blood Mini kit (Qiagen, Inc., Valencia, CA, USA) in accordance with the manufacturer's instructions. The SNP array was performed according to the manufacturer's protocols. Briefly, genomic DNA samples (250 ng) were digested, amplified and labeled. The DNA was hybridized to CytoScan HD array on the GeneChip Hybridization Oven 645 (Affymetrix, Inc., Santa Clara, CA, USA) at $50^{\circ} \mathrm{C}$ for $16-18 \mathrm{~h}$, washed on the GeneChip Fluidics Station 450 (Affymetrix, Inc.), stained with Affymetrix GeneChip Stain Reagents, and scanned on the GeneChip Scanner 3000 7G (Affymetrix, Inc.). The raw data were analyzed using Chromosome Analysis Suite 3.0 software (ChAS 3.0; Affymetrix, Inc.) The CytoScan HD array contains more than 2.6 million markers for copy number analysis; $1,950,000$ of these markers are unique, non-polymorphic oligonucleotide probes, and 750,000 markers are SNP probes used for genotyping. The average marker spacing is one probe every $1.1 \mathrm{~kb}$, with a mean spacing of one probe every $1.7 \mathrm{~kb}$ on non-gene backbones and one probe every $880 \mathrm{bp}$ in intragenic regions. Using ChAS 3.0 software (Affymetrix, Inc.), aberrations were filtered up to a minimal size of $100 \mathrm{~kb}$, with $\geq 50$ probe calls for deletions and duplications.

RefSeq and Database of Chromosome Imbalance and Phenotype in Humans Using Ensemble Resources (DECIPHER). The building of the human genome assembly was based on Homo sapiens GRCH37/hg19. The gene content of the deleted region, including Online Mendelian Inheritance in Man genes and RefSeq genes, was viewed and assessed with the UCSC Genome Browser (genome.ucsc.edu). The location and size of the deleted region was compared with similar cases described in previous CMA-based studies and the DECIPHER (decipher. sanger.ac.uk/).

\section{Results}

Routine karyotyping of the two patients revealed similar karyotypes of 46, XX, del(10) (q26) (Fig. 1A). The parents of the patients presented with normal chromosome karyotypes, demonstrating the de novo origin of the examined 10q26 deletions.

In the SNP array for Patient 1, a 10q terminal deletion with a breakpoint at 10q26.11 was detected (Fig. 1B). The deleted region was $\sim 14.04 \mathrm{Mb}$ in size (chr10: 121,385,398-135,427,143) and contained $\sim 117$ RefSeq genes ( $~ 83$ known coding genes). In the SNP array for Patient 2, the 10q terminal deletion was $\sim 13.04 \mathrm{Mb}$ in size (chr10: $122,387,570-135,427,143)$ with a breakpoint at 10q26.12 (Fig. 1B). The deleted region encompassed $\sim 108$ RefSeq genes ( 78 known coding genes). Patient 1 exhibited a larger proximally deleted region compared with Patient 2. Thus, the location of the deletion (chr10: $121,385,398-135,427,143)$ in Patient 1 was used as a search index in the DECIPHER database. The eventual results of this search indicated that the deleted regions of 30 previous cases involving 10q26 deletion were encompassed by this region (Fig. 2). These cases shared certain phenotypes with 10 q26 deletion syndrome. Furthermore, to the best of our knowledge, $\geq 18$ cases of 10q26 deletions have been characterized by CMA in prior studies (Table I). The clinical features and locations of the 10q26 deletions of patients from the current study, previous studies and the DECIPHER 
A

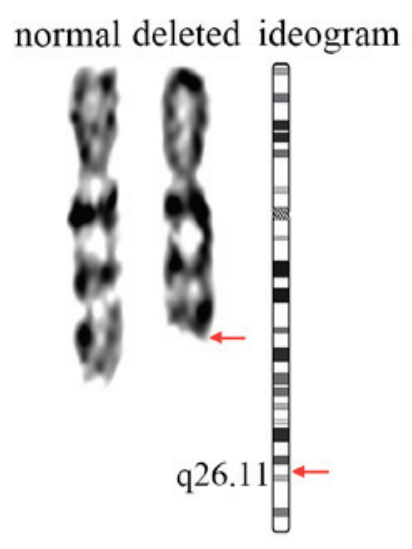

normal deleted ideogram

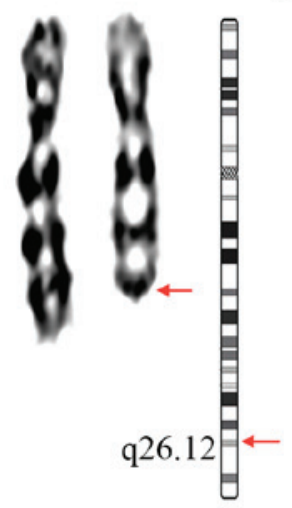

B

\author{
Patient 1 \\ Copy number state (segments)
}

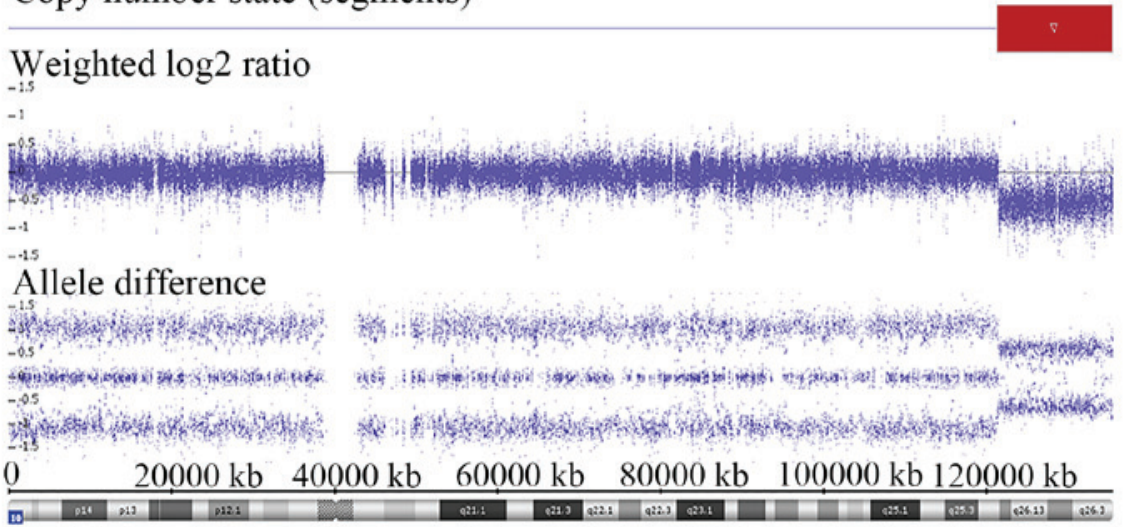

Patient 2

Copy number state (segments)

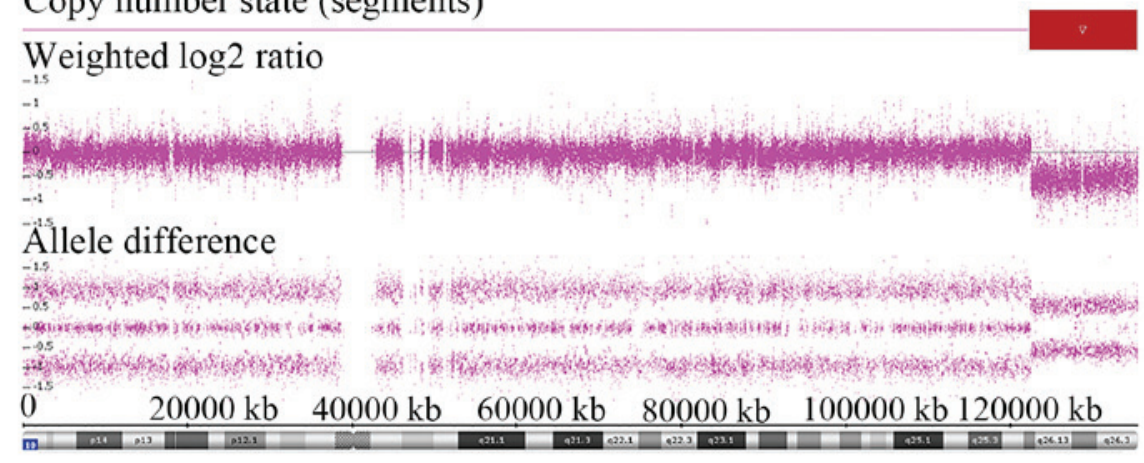

Figure 1. Results of karyotyping and SNP array analysis in Patient 1 and Patient 2. (A) Karyograms and ideograms of chromosome 10 in Patient 1 and Patient 2. Arrows indicate the locations of breakpoints. The karyotypes of both patients were 46, XX, del (10)(q26). (B) The SNP array defined a deleted genomic region from 10q26 to 10qter in the two examined patients. The findings for Patients 1 and 2 were analyzed using ChAS 3.0 software. Both $\log 2$ ratios and SNP genotyping calls accurately indicate the locations and sizes of the deleted regions. The red box outlines the deleted regions. SNP array analysis refined the results, specifically indicating that the deleted region of Patient 1 was $\operatorname{del}(10)(q 26.11)$, which spans a $14.04 \mathrm{Mb}$ segment (chr10:121,385,398-135,427,143), and that the deleted region of Patient 2 was del (10)(q26.12), which spans a 13.04 Mb segment (chr10:122,387,570-135,427,143). SNP, single nucleaotide polymorphism.

database are reviewed in Fig. 2 and Table I in an attempt to map or refine a CR.

\section{Discussion}

In the present study, the deleted regions from two patients with $10 \mathrm{q} 26$ terminal deletions were molecularly characterized using a high-resolution SNP array. This approach allowed for the accurate determination of the locations and sizes of these regions. The two patients shared various clinical features associated with 10q26 deletion syndrome, including DD/ID, growth retardation, craniofacial dysmorphism and CHDs. The findings from these patients also emphasized several of the common clinical characteristics observed in patients with pure $10 \mathrm{q} 26$ deletions.

The severity and extent of clinical characteristics associated with 10q26 deletions may depend on the varying locations and sizes of these deletions and the number of haploinsufficient genes in deleted regions. The characterization of atypical, overlapping and distinct deletions may lead to a map of CRs or even the identification of candidate genes in particular cases. Although correlations between the location and size of 10 q26 deletions and phenotypic variations remain incompletely elucidated, $\geq$ five CRs in the 10q26 region have been previously hypothesized for 10q26 deletion syndrome $(7,10,12,13)$. Yatsenko et al (7) proposed a $\sim 600 \mathrm{~kb}$ CR (Fig. 2A) at 10q26.2 for common clinical features, including craniofacial dysmorphism, CHD and DD/ID. This proposed CR contains two protein-coding genes: The chromosome 10 open reading frame 90 (C10orf90) gene and the dedicator of cytokinesis 1 (DOCK1) gene. The latter gene influences various cellular biological processes, including phagocytosis, cell migration, apoptosis and tumorigenesis, among others. The DOCK1 gene is also thought to be important in the pathogenesis of cardiovascular and urinary anomalies. Thus, Yatsenko et al (7) suggested the DOCK1 gene as a candidate gene for 10q26 deletion syndrome. The findings of the current study have further supported the idea that this proposed CR (Fig. 2A) may be important in 10q26 deletion syndrome, as the majority of the clinical features of the patients assessed in the present study were consistent with the presence of smaller deleted regions that overlap with this CR. Notably, the current study identified that 5 patients described in 
A

B

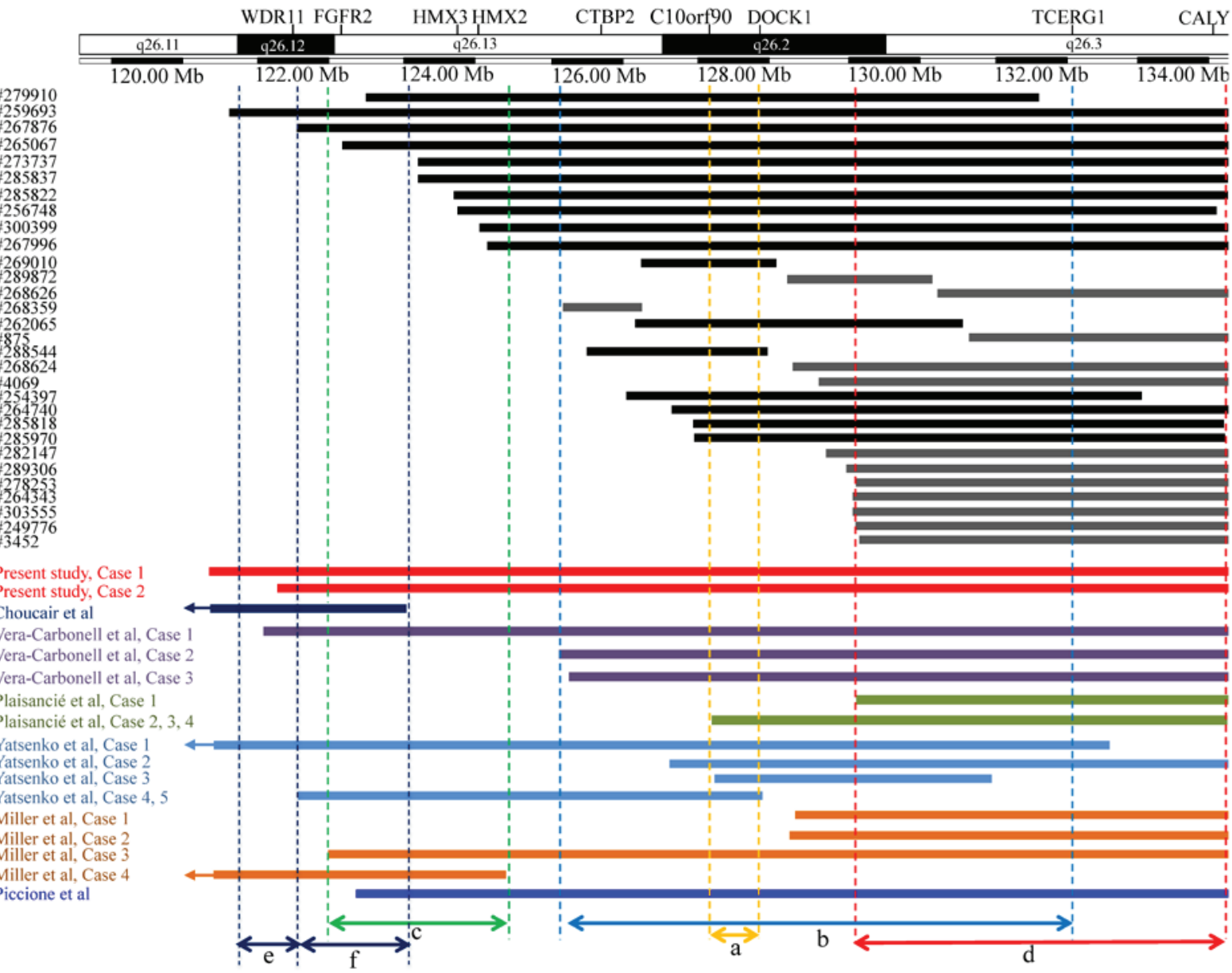

Figure 2. An overlapping map of 10q26 deletions in CMA-assessed patients described in the present study, prior studies and the DECIPHER database. (A) A schematic presentation of the 10q26 region with gene positions as specified by the University of California Santa Cruz Genome Browser. Double-headed arrows between dotted lines indicate different CRs; a=the CR proposed by Yatsenko et al (7) for common clinical features of 10q26 deletion syndrome; $\mathrm{b}=$ the CR proposed by Vera-Carbonell et al (10) for renal/urinary anomalies; c=the CR proposed by Miller et al (12) for inner ear malformation, vestibular dysfunction and hearing loss; $\mathrm{d}=$ the deleted region suggested by the current study as a region that may contribute to common clinical features of $10 \mathrm{q} 26 \mathrm{deletion}$ syndrome; e=the CR proposed by Choucair et al (13) for genital anomalies; and $\mathrm{f}=$ the CR proposed by Choucair et al (13) for common clinical features. (B) An overview of patients from the DECIPHER database with distal 10q26 deletions. Both black and gray vertical bars represent deleted regions; the black bars overlap with the CR proposed by Yatsenko et al (7), but the gray bars do not. Of 13 patients represented by gray vertical bars, 12 patients overlap with the deletion with a breakpoint at approximately $130.0 \mathrm{Mb}$ instead of the CR proposed by Yatsenko et al (7). The majority of these 12 patients presented with common features of 10q26 deletion syndrome. (C) An overview of CMA-assessed patients with 10q26 deletions described in various reports. Only four cases, including cases 1,2 and 4 described by Miller et al (12) and case 1 described by Plaisancié et al (14), exhibited common clinical features of 10q26 deletion syndrome and harbored the smallest terminal deletion with a breakpoint at approximately 130.0 Mb. CMA, chromosomal microarray analysis; CR, critical region; FGFR2, fibroblast growth factor receptor 2; CTBP2, C-terminal binding protein 2; DOCK1, dedicator of cytokinesis 1; TCERG1, transcription elongation regulator 1; CALY, calcyon neuron specific vesicular protein.

previous studies [cases 1, 2 and 4 reported by Miller et al (12) and case 1 reported by Plaisancié et al (14) and a case described by Choucair et al (13)] and 13 patients (Fig. 2B; represented by gray vertical bars) in the DECIPHER database did not have deletions that overlapped with this CR (Fig. 2A), although the majority of these patients presented with common features of 10q26 deletion syndrome. Based on the overlapping map generated in this study (Fig. 2), it was demonstrated that 15 patients, including 3 patients reported in previous studies [cases 1 and case 2 reported by Miller et al (12) and case 1 reported by Plaisancié et al (14)] and 12 patients (Fig. 2B; represented by gray vertical bars) in the DECIPHER database, overlapped with a small terminal deletion with a breakpoint at $\sim 130 \mathrm{Mb}$. However, the majority of these 15 patients presented with common features of 10q26 deletion syndrome. Therefore, it is reasonable to suspect that distal 10q26 harbors other CRs. We hypothesize that the distal 10q26 terminal deletion with a breakpoint at $\sim 130.0 \mathrm{Mb}$ may contribute to the common clinical features of 10q26 deletion syndrome.

In addition to a $\mathrm{CR}$ for common characteristics of this syndrome, other CRs for specific phenotypes are increasingly being identified. Miller et al (12) described a common $2.5 \mathrm{Mb}$ deletion region (Fig. 2C) associated with inner ear malformations, vestibular dysfunction and hearing loss in two patients. However, 7 out of 10 patients from the current and previous studies that had distal 10q26 deletions overlapping this common $2.5 \mathrm{Mb}$ region did not exhibit hearing impairment. These 7 patients included a patient described by Piccione et al (15), patients 4 and 5 in a report by Yatsenko et al (7), patient 1 in a report by Vera-Carbonell et al (10), a patient described by Choucair et al (13) and the two patients described in the present report. A similar trend is observed for all cases 


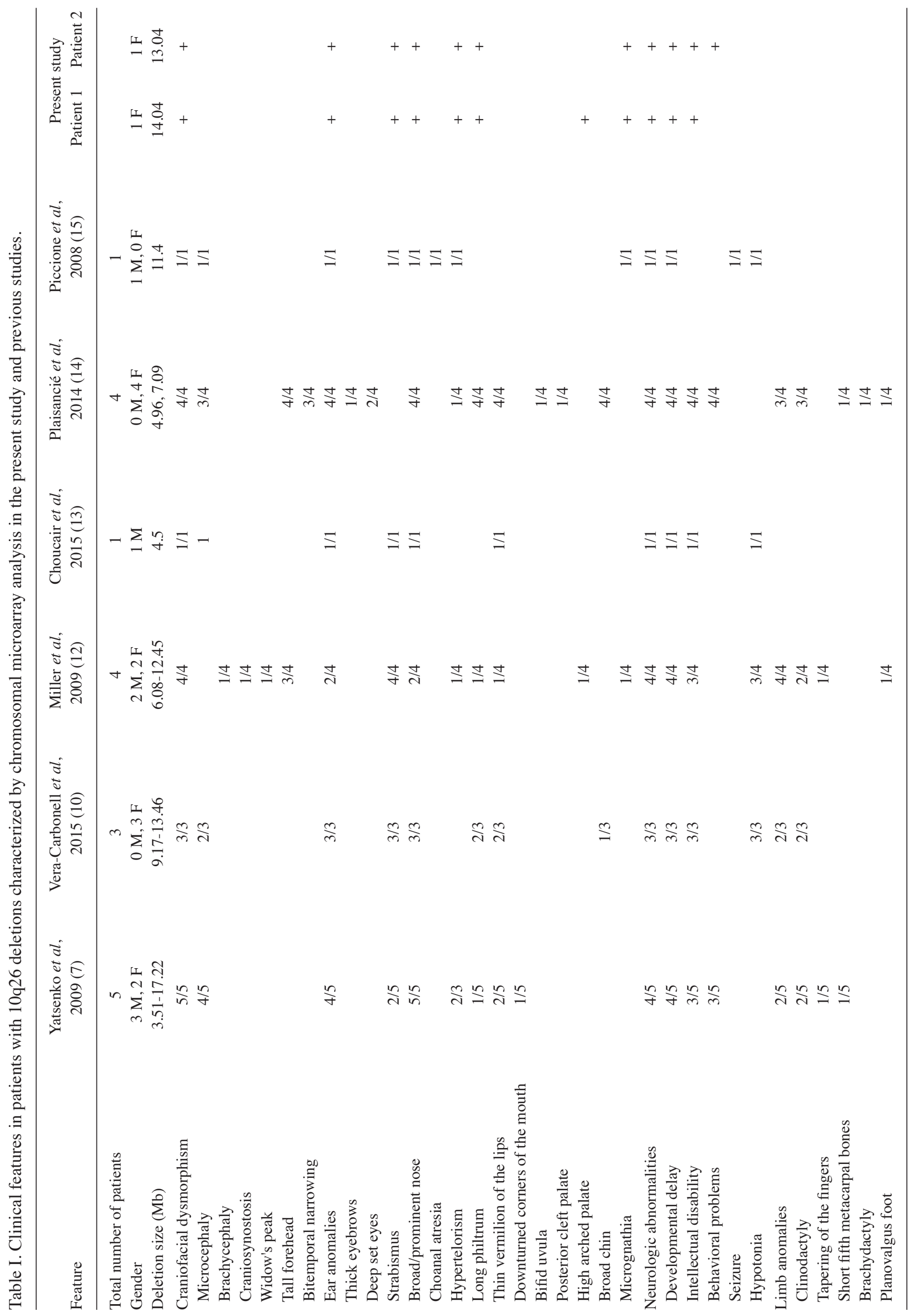




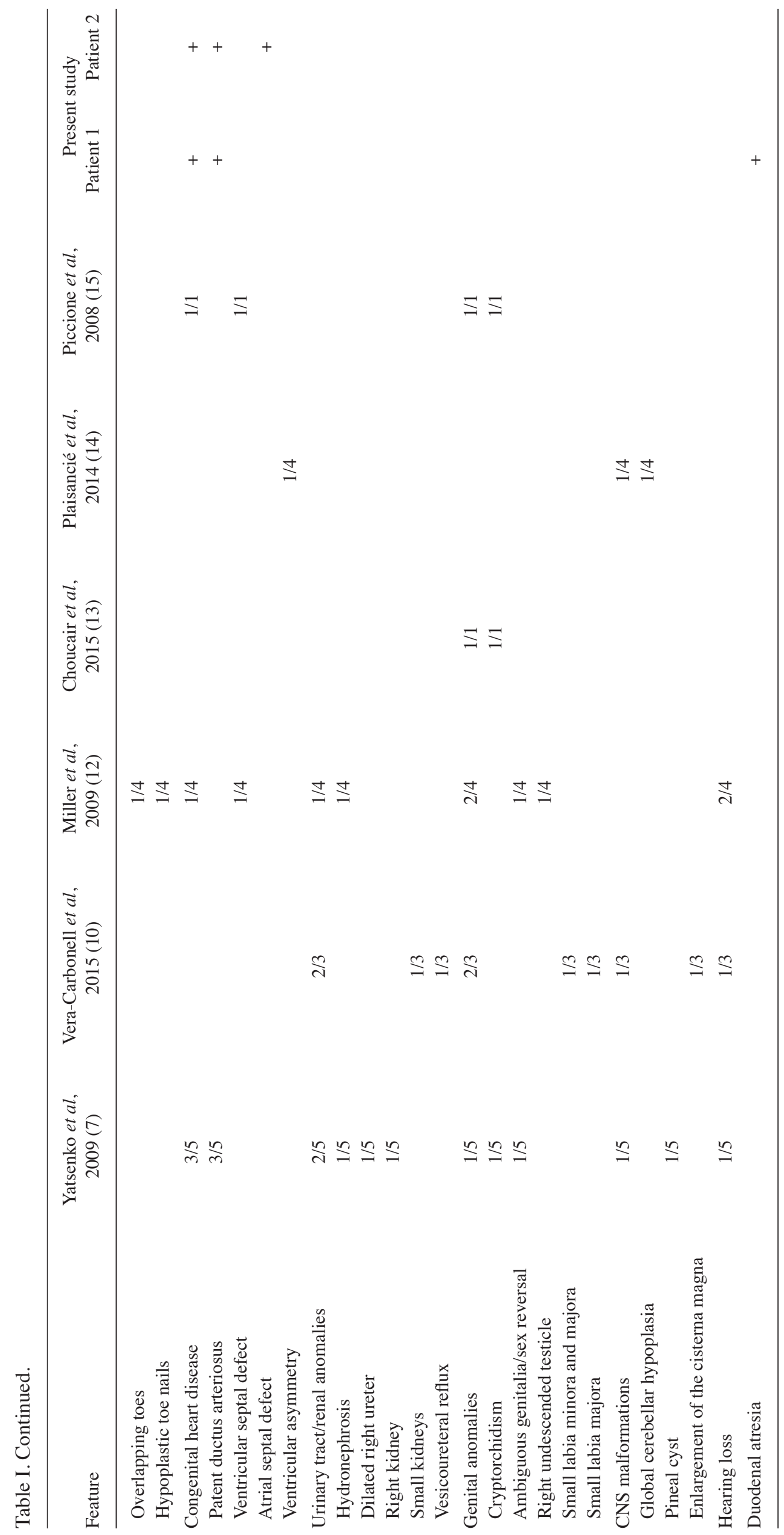


recorded in the DECIPHER database that involve a deletion in this region. Notably, among three patients described by Vera-Carbonell et al (10), patient 3 did not harbor a deletion that overlapped with this region, but did exhibit hearing loss, patient 2 did not harbor a deletion that overlapped with this region or present with hearing loss, and patient 1 exhibited normal hearing, in spite of harboring a deletion in this region. In the previous study, Vera-Carbonell et al (10) focused on renal/urinary tract anomalies associated with 10q26 deletions and suggested that small 10q26.2 distal deletions may be associated with common phenotypic characteristics rather than renal/urinary tract anomalies, which are instead correlated with longer 10q26.2 distal deletions (Fig. 2B). Notably, among individuals with a 10q26 deletion overlapping the proposed CR for renal/urinary tract anomalies (Fig. 2B), 13 out of 18 patients [not including patient 4 in a report by Miller et al (12) and a case reported by Choucair et al (13)] examined in the present study and previous studies, and 24 out of 30 cases recorded in the DECIPHER database, did not exhibit such anomalies. Recently, Choucair et al (13) also proposed a novel CR for genital abnormalities (Fig. 2E) and another different CR for common clinical features (Fig. 2F). Through a review of the present study and previous studies, it was observed that genital abnormalities were presented in 4 out of 6 cases with a 10q26 deletion overlapping the above proposed CR for genital abnormalities (Fig. 2E). However, genital abnormalities were not observed in the two cases of the current study. In addition to potential CRs, studies of 10q26 deletions have also proposed candidate genes, including the $D O C K 1$, fibroblast growth factor receptor 2 (FGFR2), C-terminal binding protein 2 (CTBP2), calcyon neuron specific vesicular protein $(C A L Y)$, WD repeat domain 11 (WDR11), H6 family homeobox 2 (HMX2) and $H M X 3$ genes within these regions $(7,10,12-14)$. Phenotypic effects correlated with CRs may primarily depend on the number of haploinsufficient genes in deleted regions. However, to the best of our knowledge, there is little available evidence to confirm the pathogenicity of haploinsufficiency for either candidate genes or other genes in the $10 q 26$ region. Further investigations should be performed to identify dosage-sensitive genes in this region and thereby obtain an improved understanding of the genotype-phenotype correlations associated with 10q26 deletions.

The conflicting findings discussed above may be attributed to incomplete penetrance/variable expressivity or the effects of other potential CRs. Furthermore, if potential CRs were assumed to be independent genomic sites, a 'two-hit' model would account for these discordances. This model would propose that the combined effects of two or more deletions would result in phenotypic variability $(16,17)$. Each CR may contribute to particular phenotypes in a correlative manner; as a result, manifestations of the clinical characteristics of $10 \mathrm{q} 26$ deletion syndrome would be expected to be variable or incomplete in the patients described in these studies.

In conclusion, the current study reported two new patients with 10q26 deletion syndrome and reviewed the literature regarding this syndrome. Although an overlap map for CMA-assessed cases from the present study, previous studies and the DECIPHER database, was generated CR refinement could not be accomplished due to the limited number of available cases and the varying and atypical deletions observed in these cases. However, in addition to the CR suggested by
Yatsenko et al (7), the results of the current study suggest that the distal 10q26 terminal deletion with a breakpoint at $\sim 130.0 \mathrm{Mb}$ may also contribute to common clinical features of 10q26 deletion syndrome. In addition, the association between three previously reported CRs and phenotypic variability were discussed in detail, thus, facilitating improved comprehension of the phenotypic heterogeneity of 10q26 deletion syndrome.

\section{References}

1. Lewandowski RC Jr, Kukolich MK, Sears JW and Mankinen CB: Partial deletion 10q. Hum Genet 42: 339-343, 1978.

2. Wulfsberg EA, Weaver RP, Cunniff CM, Jones MC and Jones KL: Chromosome 10qter deletion syndrome: A review and report of three new cases. Am J Med Genet 32: 364-367, 1989.

3. Schrander-Stumpel C, Fryns JP and Hamers G: The partial monosomy 10q syndrome: Report on two patients and review of the developmental data. J Ment Defic Res 35: 259-267, 1991.

4. Irving M, Hanson H, Turnpenny P, Brewer C, Ogilvie CM, Davies A and Berg J: Deletion of the distal long arm of chromosome 10; is there a characteristic phenotype? A report of $15 \mathrm{de}$ novo and familial cases. Am J Med Genet A 123A: 153-163, 2003.

5. Courtens W, Wuyts W, Rooms L, Pera SB and Wauters J: A subterminal deletion of the long arm of chromosome 10: A clinical report and review. Am J Med Genet A 140: 402-409, 2006.

6. Scigliano S, Gregoire MJ, Schmitt M, Jonveaux PH and LeHeup B: Terminal deletion of the long arm of chromosome 10. Clin Genet 65: 294-298, 2004.

7. Yatsenko SA, Kruer MC, Bader PI, Corzo D, Schuette J, Keegan CE, Nowakowska B, Peacock S, Cai WW, Peiffer DA, et al: Identification of critical regions for clinical features of distal 10q deletion syndrome. Clin Genet 76: 54-62, 2009.

8. Kehrer-Sawatzki H, Daumiller E, Müller-Navia J, Kendziorra H, Rossier E, du Bois G and Barbi G: Interstitial deletion del(10) (q25.2q25.3 approximately 26.11)-case report and review of the literature. Prenat Diagn 25: 954-959, 2005.

9. Miller DT, Adam MP, Aradhya S, Biesecker LG, Brothman AR, Carter NP, Church DM, Crolla JA, Eichler EE, Epstein CJ, et al: Consensus statement: Chromosomal microarray is a first-tier clinical diagnostic test for individuals with developmental disabilities or congenital anomalies. Am J Hum Genet 86: 749-764, 2010.

10. Vera-Carbonell A, López-Gonzalez V, Bafalliu JA, Ballesta-Martínez MJ, Fernández A, Guillén-Navarro E and López-Expósito I: Clinical Comparison of 10q26 overlapping deletions: Delineating the critical region for urogenital anomalies. Am J Med Genet A 167A: 786-790, 2015.

11. Claussen U, Michel S, Mühlig P, Westermann M, Grummt UW, Kromeyer-Hauschild K and Liehr T: Demystifying chromosome preparation and the implications for the concept of chromosome condensation during mitosis. Cytogenet Genome Res 98: 136-146, 2002.

12. Miller ND, Nance MA, Wohler ES, Hoover-Fong JE, Lisi E, Thomas GH and Pevsner J: Molecular (SNP) analyses of overlapping hemizygous deletions of 10q25.3 to 10qter in four patients: Evidence for HMX2 and HMX3 as candidate genes in hearing and vestibular function. Am J Med Genet A 149A: 669-680, 2009.

13. Choucair N, Abou Ghoch J, Fawaz A, Mégarbané A and Chouery E: 10q26.1 Microdeletion: Redefining the critical regions for microcephaly and genital anomalies. Am J Med Genet A 167A: 2707-2713, 2015.

14. Plaisancié J, Bouneau L, Cances C, Garnier C, Benesteau J, Leonard S, Bourrouillou G, Calvas P, Vigouroux A, Julia S and Bieth E: Distal 10q monosomy: New evidence for a neurobehavioral condition? Eur J Med Genet 57: 47-53, 2014.

15. Piccione M, Antona V, Piro E, Cavani S, Malacarne M, Pierluigi $\mathrm{M}$ and Corsello G: 10qter deletion: A new case. Am J Med Genet A 146A: 2435-2438, 2008.

16. Girirajan S, Rosenfeld JA, Coe BP, Parikh S, Friedman N, Goldstein A, Filipink RA, McConnell JS, Angle B, Meschino WS, et al: Phenotypic heterogeneity of genomic disorders and rare copy-number variants. N Engl J Med 367: 1321-1331, 2012.

17. Girirajan S, Rosenfeld JA, Cooper GM, Antonacci F, Siswara P, Itsara A, Vives L, Walsh T, McCarthy SE, Baker C, et al: A recurrent 16p12.1 microdeletion supports a two-hit model for severe developmental delay. Nat Genet 42: 203-209, 2010. 